Rechtsmedizin 2018 · 28:249-250 https://doi.org/10.1007/s00194-018-0265-3 Online publiziert: 27. Juni 2018

(c) Springer Medizin Verlag $\mathrm{GmbH}$, ein Teil von Springer Nature 2018

CrossMark

\section{R. Lessig}

Institut für Rechtsmedizin, Universitätsklinikum Halle, Halle (Saale), Deutschland

Halle/Saale, 12.-15.09.2018

Sehr geehrte Mitglieder der Deutschen Gesellschaft für Rechtsmedizin, liebe Kolleginnen und Kollegen,

in der Zeitschrift Rechtsmedizin werden traditionell die Abstracts zu den Vorträgen und Posterbeiträgen der anstehenden Jahrestagung veröffentlicht. Schon an dieser Stelle soll allen gedankt sein, die mit ihren Arbeiten zum Gelingen beitragen werden. Wir freuen uns, dass so viele Beitragsanmeldungen eingegangen sind. Eine Neuerung ist in diesem Jahr am Samstag, den 15.09.2018, zu verzeichnen. An diesem Tag wird erstmals der interdisziplinäre Arbeitskreis für Forensische Odonto-Stomatologie (AKFOS) seine Jahrestagung gemeinsam mit der DGRM abhalten.

Die Mitarbeiter des Instituts für Rechtsmedizin der Martin-Luther-Universität Halle-Wittenberg freuen sich, dass Sie so zahlreich nach Sachsen-Anhalt, speziell nach Halle an der Saale, kommen. Sachsen-Anhalt als Ursprungsland der Reformation hat dieses Ereignis im vergangenen Jahr im großen Rahmen gefeiert. Mit entsprechendem Stolz trägt die Universität, die aus dem Zusammenschluss zwischen der Wittenberger Universität Leucorea (gegr. 1502) und der Friedrichs-Universität Halle (gegr. 1694) im Jahr 1817 auf Veranlassung von Friedrich III. Markgraf von Brandenburg und Kurfürst des Heiligen Römischen Reiches und souveräner Herzog in Preu- ßen hervorgegangen ist, den Namen des Reformators. Die 200-Jahr-Feier zur Universitätsfusion fand ebenfalls im letzten Jahr statt. Halle ist aber nicht nur Standort der Universität, sondern auch der Deutschen Akademie der Wissenschaften, der Leopoldina und der Kunsthochschule Burg Giebichenstein, hoch über der Saale gelegen. Der Begrüßungsabend wird in der Leopoldina, dem Weißen Haus von Halle, stattfinden. Auch ist ein Ausflug in die Weinanbauregion von Sachsen-Anhalt für den Freitagnachmittag geplant. Dieser geht zunächst in die Domstadt Naumburg und anschließend zum Gesellschaftsabend in die Sektkellerei Rotkäppchen nach Freyburg.

\section{》) Das Tagungsmotto „Vom Universalgelehrten Alberti zum forensischen Spezialisten" spiegelt die Geschichte des Faches wider}

Der im Zentrum der Stadt gelegene historische Universitätscampus bietet die Möglichkeit, unsere Jahrestagung dort abzuhalten, und somit kurzer Wege zwischen Tagungsort und Stadtzentrum. Das Motto der Tagung ist ,Vom Universalgelehrten Alberti zum forensischen Spezialisten“. Das Thema soll die enge Beziehung zwischen der juristischen
Fakultät und der Rechtsmedizin in Halle, aber auch die Geschichte des Fachs widerspiegeln. Spezielle Obduktionsbefunde, die erstmals in Sachsen-Anhalt bzw. Halle beschrieben wurden, sind heute fester Bestandteil der Standards in unserem Fach. Entsprechend dem Motto der Tagung sind die eingereichten Beiträge breit gefächert und spiegeln das gesamte Spektrum des Fachs wider. Die Tatsache, dass einer der eingeladenen Redner aus der juristischen Fakultät der hiesigen Universität kommt, unterstreicht die bestehenden engen Beziehungen zwischen der Rechtsmedizin und den Juristen. Die Existenz des MasterStudiengangs Medizin - Ethik - Recht, bei dessen Gründung die Rechtsmedizin schon ein wesentlicher Bestandteil war, ist ein Beispiel für die sehr lebendigen Beziehungen in Halle.

Die Rechtsmedizin hat in den letzten Jahren immer auch im Fokus der Politik und somit der Landesregierung von Sachsen-Anhalt gestanden. Der Ausschreibung nur noch eines Lehrstuhls in Sachsen-Anhalt, dem in Halle, folgte ein Beschluss zur Fusionierung der Institute von Halle und Magdeburg. Der Verzicht auf den Lehrstuhl Magdeburg ist sehr bedauerlich gewesen, da dort in den Jahren zuvor herausragende Forschung, insbesondere auf dem Gebiet der Molekulargenetik, erfolgte. Letztlich muss aber auch festgestellt werden, dass in der politischen Diskussion immer die 
Bedeutung des Fachs für die Strafverfolgungsbehörden herausgehoben wurde. Dabei wurde auch die Tätigkeit auf dem Gebiet des Gewaltopferschutzes gewürdigt. Die in den letzten Jahren stark gestiegenen Zahlen der Gewaltopferuntersuchungen zeigen die Notwendigkeit einer rechtsmedizinischen Versorgung nicht nur in den großen Kliniken der Städte, sondern auch in der Fläche. Es muss auch erwähnt werden, dass im Land Sachsen-Anhalt ein Gesetz zum Kinderschutz lange vor der Etablierung des diesbezüglichen Bundesgesetzes existierte. Das Gesetz wurde durch eine vom Ministerium für Arbeit, Soziales und Integration etablierte Allianz für Kinderschutz ergänzt. Diese hat 2015 einen aktualisierten Leitfaden zu diesem Thema herausgegeben. Die Landesregierung stellte in den letzten Jahren zusätzliche finanzielle Mittel zur Sicherstellung der Arbeitsfähigkeit des Instituts zur Verfügung. Dadurch war es möglich, eine Außenstelle in Magdeburg, die für die dortige medizinische Fakultät unverzichtbar ist, zu erhalten und die zeitnahe Versorgung des nördlichen Sachsen-Anhalt zu gewährleisten. Hoffen wir, dass auch die Jahrestagung dazu beiträgt, die positive Entwicklung aufrechtzuerhalten.

Ihr

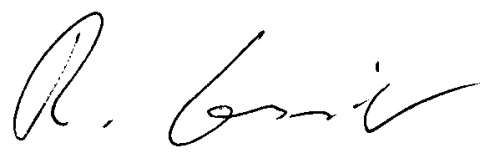

Rüdiger Lessig

\section{Korrespondenzadresse}

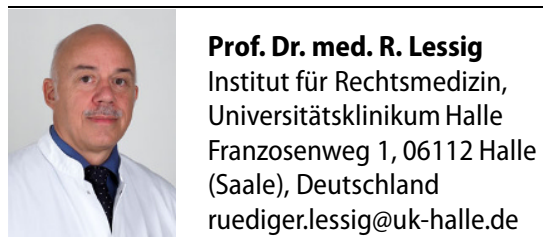

Interessenkonflikt. R. Lessig gibt an, dass kein Interessenkonflikt besteht.

Hans Joseph Böhles, Mayyada Qirshi

\section{Transkulturelle Medizin}

Migranten aus muslimischen und afrikanischen Lebenswelten im ärztlichen Alltag

Heidelberg Berlin: Springer-Verlag 2018, 1. Aufl., 188 S., 25 Abb., (ISBN: 978-3-66256034-1), Softcover 39,99 EUR, + e.Book inside

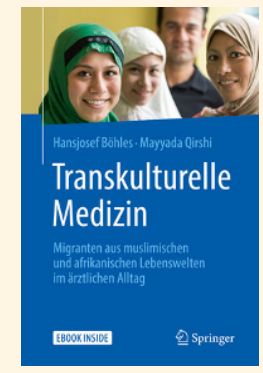

Der ehemalige Direktor der Universitäts-Kinder- und Jugendklinik Frankfurt am Main H.J.Böhles und die im Jemen geborene Kinderärztin M. Qirshi haben ein lesenswertes, aktuell zur Wissensvermittlung erforderliches und informatives Werk zur transkulturellen Medizin vorgelegt.

Das Buch ist ein Ratgeber für Ärzte und medizinisches Personal, die mit der Behandlung und Betreuung von Migranten aus muslimischen und afrikanischen Ländern betraut sind. Berücksichtigt werden auch asiatische Migranten und Besonderheiten bei mehreren Migrantengenerationen.

Ein Hauptanliegen der Autoren ist die Vermittlung von Kenntnissen über den Einfluss von Herkunftskultur und Religion (Islam, Religion der Jesiden, Judentum, Religionen Afrikas) auf das Verhalten von vorgestellten Patienten und deren Angehörigen in Praxis und Klinik. Dabei werden kulturtypische Besonderheiten herausgearbeitet, wie Bekleidung, Barttracht bei Männern, Tattoos, Gesten, Signale und Farben sowie Sprachbesonderheiten und Fremdsprachenkenntnisse in ehemaligen Kolonialgebieten, ebenso Spezifika von Geburt, Ehe und Tod.

Eingegangen wird weiterhin auf Kommunikationsformen und mögliche Missverständnisse bei Patientenvorstellungen, wie den für Migranten wichtigen „Wohlfühlabstand“, typische Formen der Geschlechterbegegnung, kulturtypische jüdische und muslimische Ernährungsformen, kulturtypische Eigenheiten gegenüber Präventionsmaßnahmen wie die Einstellung zu Impfungen, therapeutische Hautreizungen, die Nutzung von Muttermilch zur Hautpflege, die Bevorzugung von Injektionen und die Ablehnung von Zäpfchen, auf die rituelle Abwendung des „Bö- sen Blicks" u.a. Auch werden kulturtypische Suchtformen und spezielle Untersuchungsbefunde (Besonderheiten der Haut, rituelle Eingriffe am Genitale u.a.) aufgezeigt und ihrer Bedeutung gemäß bewertet.

Spezielle Kapitel sind darüber hinaus den häufigsten Erkrankungen von Migranten gewidmet, unter andern Infestationen und Infektionen wie Malaria, Gelbfieber und das häufige Denguefieber, der Tuberkulose und der HIV- Infektion, der Zahngesundheit, den Haut- und hämatologischen Erkrankungen (hier speziell der Thalassämie und Sichelzellanämie) sowie metabolischen und immunologischen Problemen, wie dem Diabetes mellitus und dem Mittelmeerfieber.

Die gestrafften klaren Krankheitsbeschreibungen umfassen 65 der 182 Textseiten. Der Schwerpunkt des Buches liegt auf der für das ärztliche Handeln so wichtigen Vermittlung von interkulturellen Charakteristika.

Das Werk ist großzügig ausgestattet, hat eine adäquate Bild-Text-Relation und verfügt über ein „eBook Inside“. Das Verständnis der zum überwiegenden Teil prägnanten Ausführungen wird durch eingefügte Merksätze und praktische Hinweise zusätzlich erleichtert und gefördert. Das Literaturverzeichnis befindet sich auf dem aktuellen Stand (Literaturnachweise bis 2017).

Das Buch kann allen mit der medizinischen Betreuung von Patienten aus muslimischen und afrikanischen Ländern befassten Berufsgruppen - Ärzten Schwestern, Psychologen, Sozialarbeitern und Therapeuten verschiedener Fachgruppen - sowie weiteren Interessenten besten empfohlen werden.

V. Hesse (Berlin) 